\title{
GPPS-BJ-2019-0099
}

\section{An Analytical Approach to Estimate the Effect of Surface Roughness on Particle Rebound}

\author{
Johannes Altmeppen \\ Institute of Aircraft Propulsion \\ University of Stuttgart \\ johannes.altmeppen@ila.uni-stuttgart.de \\ Stuttgart, Germany \\ Christian Koch \\ Institute of Aircraft Propulsion \\ University of Stuttgart \\ christian.koch@ila.uni-stuttgart.de \\ Stuttgart, Germany
}

\author{
Heike Sommerfeld \\ Institute of Aircraft Propulsion \\ University of Stuttgart \\ heike.sommerfeld@ila.uni-stuttgart.de \\ Stuttgart, Germany
}

\author{
Stephan Staudacher \\ Institute of Aircraft Propulsion \\ University of Stuttgart \\ stephan.staudacher@ila.uni-stuttgart.de \\ Stuttgart, Germany
}

\begin{abstract}
Atmospheric air is always contaminated by liquid or solid particles of different size, concentration and chemical composition. This leads to performance degradation during the operation of stationary or flying gas turbines. Erosion and the deposition of particles along the flow path are of particular importance. Multiple numerical studies investigated the influences of these phenomena. However, the basic challenge of modelling the particle wall interaction and its data spread with sufficient accuracy remains.

In this work a model that estimates the statistical spread of rebound data due to target surface roughness through analytical considerations is presented. The model predicts the local impact angle of an individual particle by evaluating how deep a particle can theoretically penetrate the target surface with respect to its size. Based on roughness profiles which have been found to be characteristic for performance deterioration in compressor application a sensitivity study is conducted. A dimensionless roughness parameter $\Phi_{R}$ was found that characterizes the effect of target surface roughness on rebound spread data. The spread model is connected with a quasi-physical model by (Bons et al., 2017), to evaluate the effect of surface roughness for a particle's individual rebound behaviour. The synthesized data is discussed by taking into account measurement data reported in literature.
\end{abstract}

\section{INTRODUCTION}

Aircraft engines are exposed to a variety of external influences during operation causing gradual engine deterioration and damages over operation time (Kellersmann et al., 2018). Main influences concerning the engines high pressure compressors are deposition and erosion due to airborne particles (Richardson et al., 1979). To predict realistic erosion as well as deposition patterns, accurate modelling of the particle rebound after impacting a surface is indispensable.

Particle rebounds are statistical in nature due to varying particle sizes, particle shapes, target surface topography, particle rotation and particle break-up during contact. Existing modelling approaches based on empirical correlations fail to predict the spread in the coefficients of restitution (CoR) at different operating conditions than the original experimental setup by up to $100 \%$ (Bons et al., 2015). Furthermore, present modelling approaches based on physical considerations have focused on modelling particle impacts mean rebound coefficients only. Standard deviations of the coefficients of restitution vary with the considered particle and target surface combination. Hence excessive experimental testing of different particle-surface combinations is needed to simulate the statistical spread numerically. The scope of the presented work is to access the statistical spread of particle rebound data through analytical considerations. For simplification only statistical spread of particle rebound due to target roughness will be discussed here as a first step towards a general physical based spread model. The presented approach is based on work of Sommerfeld and Huber (Sommerfeld and Huber, 1999). It derives local impact angles $\alpha_{1}^{\prime}$ due to surface roughness for individual particles, which may deviate by $\Delta \alpha$ from the global impact angle $\alpha_{1}$ as shown in Figure 1. It can be combined with an existing rebound model like the one presented by (Bons et al., 2017) (Figure 2). 
Further input variables for the Bons-Model are used according to (Whitaker and Bons, 2018) for ARD particles in a $1-100 \mu \mathrm{m}$ size distribution.

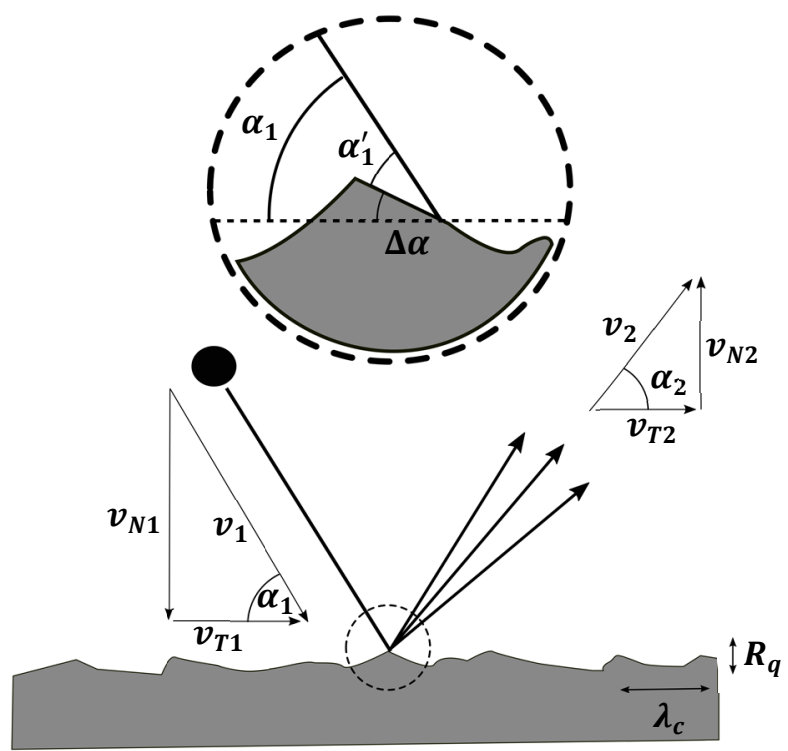

Figure 1: Determination of velocities and angles before and after impact

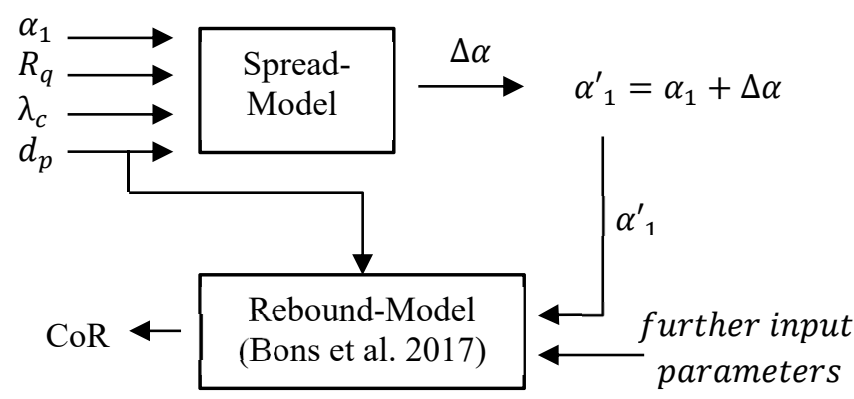

Figure 2: Diagram of model implementation in a closed modelling loop

\section{THEORETICAL BACKGROUND}

Usually coefficients of restitution are used to describe the energy loss during collision. They are defined as the magnitude of a parameter after rebound divided by its corresponding magnitude at impact (Figure 1):

$$
\operatorname{CoR}_{v}=\frac{v_{2}}{v_{1}} ; \operatorname{CoR}_{\alpha}=\frac{\alpha_{2}}{\alpha_{1}} ; \operatorname{CoR}_{N}=\frac{v_{N 2}}{v_{N 1}} ; \operatorname{CoR}_{T}=\frac{v_{T 2}}{v_{T 1}}
$$

To estimate these coefficients in turbomachine applications, rebound and deposition models are used. They are based either on a critical velocity approach derived by Brach and Dunn (Brach and Dunn, 1992) or a critical viscosity approach based on work of Tafti and Screedharen (Sreedharan and Tafti, 2010). Critical viscosity models concern particle softening mechanisms at high temperature applications in the turbine section and are not reviewed in this study aiming to evaluate particle impact in the engines compressors section. Deposition models based on critical velocity determine whether a particle sticks to the surface by comparing the actual impact velocity with a capture velocity for the impact event. Below this capture velocity the particle will deposit. If a particle does not deposit an estimate of the rebound velocity is applied.

These models are based on contact mechanics with elastic deformation in a Hertzian contact of a spherical particle with a flat surface in normal direction combined with adhesion during impact (Brach and Dunn, 1992). This approach has been extended to account for oblique impacts, elastic-plastic deformation and further external forces (Barker et al., 2017; Singh and Tafti, 2015; Kim and Dunn, 2007). Still it is valid for spherical particles only and rely heavily on empirical correlation (Bons et al., 2017).

Therefore Bons et al. (Bons et al., 2017) derived a quasiphysical but yet equally empirical and approximate modelling approach based on representing arbitrary particle shapes as a circular cylinder. It allows to model particle deformation as a $1 \mathrm{D}$ spring rather than a complex $3 \mathrm{D}$ deformation of a sphere. The model comprises elastic and plastic deformation, adhesion, and shear removal. It has been further improved by Whitaker (Whitaker and Bons, 2018) to account for stochastic spread of rebound data with an empirical curve fitting approach based on experimental data. Since this approach still relies on excessive experimental testing of different material and particle combinations a physical based access to the statistical spread due to surface roughness is desired in this work. Based on roughness profiles which have been found to be characteristic for performance deterioration in compressor applications (Table 1) a sensitivity study has been conducted. The results of the sensitivity study will be discussed after introducing the developed modelling approach to statistical spread.

\begin{tabular}{|c|c|c|c|c|c|c|}
\hline \# & Region & Process & $\begin{array}{c}\mathrm{Ra} \\
(\mu m)\end{array}$ & $\begin{array}{c}\mathbf{R q} \\
(\mu m)\end{array}$ & Sk & $\begin{array}{c}\lambda_{c} \\
(\mu m)\end{array}$ \\
\hline 1 & $\mathrm{PS} / \mathrm{MS} / \mathrm{MC}$ & Deposits & 2.6 & 3.4 & 0.17 & 169 \\
\hline 2 & SS/MS/LE & Deposits & 2.7 & 4.9 & 1.43 & 131 \\
\hline 3 & SS/Tip/TE & Deposits & 7 & 8.9 & 0.33 & 60 \\
\hline 4 & PS/MS/LE & Deposits & 7.1 & 10.5 & 2.18 & 140 \\
\hline 5 & $\mathrm{SS} / \mathrm{MS} / \mathrm{LE}$ & Deposits & 14.1 & 19 & 0.5 & 314 \\
\hline 6 & $\mathrm{SS} / \mathrm{MS} / \mathrm{MC}$ & Deposits & 3.7 & 4.8 & 0.72 & 408 \\
\hline 7 & $\mathrm{SS} / \mathrm{MS} / \mathrm{LE}$ & Erosion \& deposits & 6.5 & 8.2 & -0.11 & 242 \\
\hline 8 & $\mathrm{SS} / \mathrm{Hub} / \mathrm{LE}$ & Erosion \& deposits & 4.4 & 5.5 & -0.3 & 114 \\
\hline 9 & $\mathrm{PS} / \mathrm{Hub} / \mathrm{TE}$ & Erosion \& deposits & 9 & 11.2 & 0.03 & 69 \\
\hline 10 & PS/Tip/TE & Erosion \& deposits & 15.1 & 19.7 & 0.03 & 208 \\
\hline 11 & SS/MS/TE & Pitting \& erosion & 7.5 & 10.7 & -1.4 & 650 \\
\hline 12 & PS/MS/TE & Pitting & 4.2 & 7.1 & -3.1 & 209 \\
\hline 13 & $\mathrm{PS} / \mathrm{MS} / \mathrm{MC}$ & Erosion & 1.9 & 2.4 & -0.11 & 372 \\
\hline 14 & SS/Tip/LE & Erosion & 4.4 & 5.9 & 0.02 & 307 \\
\hline 15 & SS/MS/TE & Clean surface & 1.1 & 1.3 & -0.4 & 170 \\
\hline
\end{tabular}

Table 1: Representative surface statistical data grouped by roughness-generating process

(Bons et al., 2001) 


\section{SURFACE ROUGHNESS MODELLING}

Based on the data exhibited in Table 1 a Design of Experiments (DoE) has been carried out:

$$
\begin{array}{ll}
\mathrm{R}_{\mathrm{q}}(\mu \mathrm{m}): & 2,5,10,15,20,25 \\
\lambda_{\mathrm{c}}(\mu \mathrm{m}): & 40,80,120,240,360,400 \\
d_{P}(\mu \mathrm{m}): & 1,2,5,10,15 \ldots 400
\end{array}
$$

The roughness root mean squared $R_{q}$ and correlation length $\lambda_{c}$ were used to characterize roughness height and elongation of the examined surface (Figure 3). The roughness profiles were processed with a morphological filter routine based on the closing principle (Serra and Vincent, 1992). The filtered profile defines how deep a particle of size $d_{P}$ impacting normal to the surface $\left(90^{\circ}\right)$ can penetrate the roughness structure (Figure 3).

The roughness profiles have been generated artificially using the methods outlined by Garcia and Stoll (Garcia and Stoll, 1984). This procedure utilises roughness profiles with different $R_{q}$ and $\lambda_{c}$ that fulfil the Gaussian statistics. Corresponding slope angles are obtained by applying the morphological filter as described above. In this way the filtered profile leads to the local impact angle $\alpha_{1}^{\prime}$, where the particle hits the surface. By applying a kernel density estimation on the sampled data a distribution function of possible impact angles has been gained. These probability distributions of the local impact angles can be approximated with a Normal Gaussian Distribution (Sommerfeld and Huber, 1999 ) with the expected value $\mu$ and the variance $\sigma^{2}$. Such an approximation provides a significant saving in required storage space in the modelling process.

The Kullback-Leibler divergence is used to evaluate the quality of the approximation (Kullback, 1978). For values $D_{K L}$ close to zero, sufficient accuracy has been archived.

$$
D_{K L}\left(\mathbf{P}_{\text {ref }}|| \mathrm{P}\right)=-\sum_{x \in X} \boldsymbol{P}_{\text {ref }}(x) \log \left(\frac{P(x)}{P_{\text {ref }}(x)}\right)
$$

Figure 4 shows an example for the kernel density estimation and its corresponding normal Gaussian approximation. The Kullback-Leibler divergence for all generated profiles are close to zero with a median value of $D_{\text {KLmedian }}=0.0251$. Hence sufficient accuracy by the approximation with a Normal Gaussian distribution has been archived.

In order to validate the whole roughness generation process a surface roughness of a deteriorated compressor blade has been scanned with an optical profilometer (Talysurf CCI - Taylor Hobson) (Casari et al., 2018) and compared with an artificially created profile of the same roughness characteristics $\mathrm{R}_{\mathrm{q}}$ and $\lambda_{\mathrm{c}}$ (Figure 5). With a value of $D_{K L}\left(P_{\text {real }} \| P_{\text {generated }}\right)=0.058$, the generated profile reproduces the slope angle distribution of the original profile with sufficient accuracy.
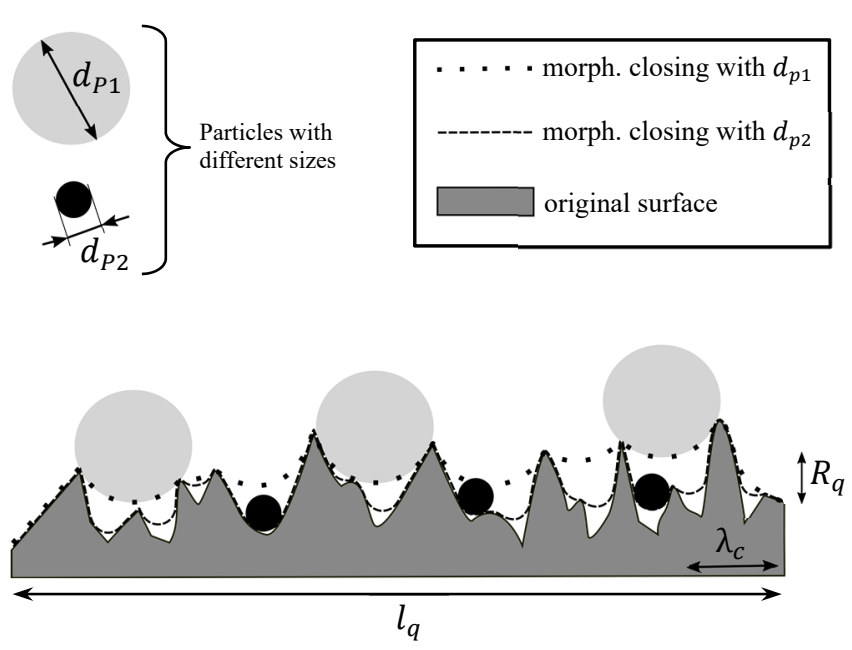

Figure 3: Roughness topography and morphological filter

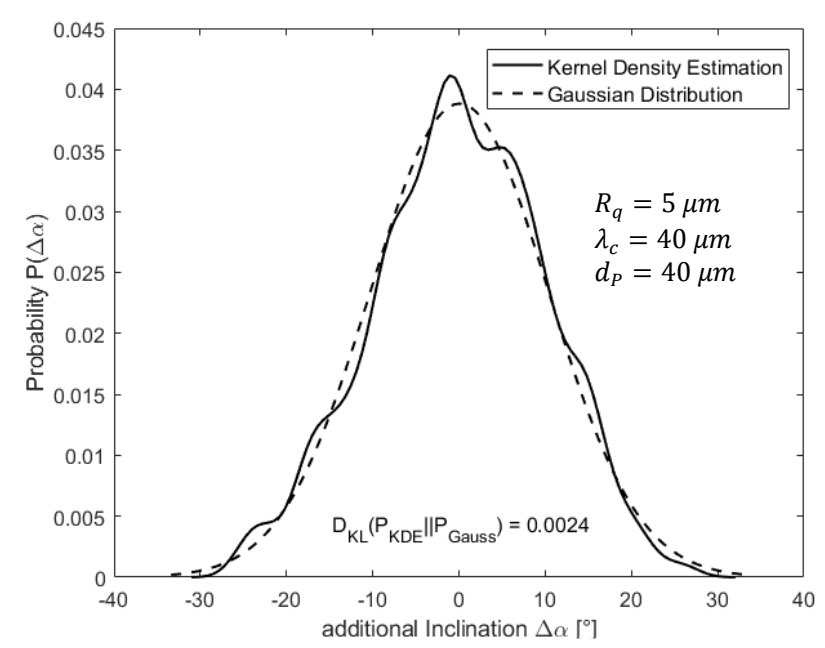

Figure 4: Quality of approximation
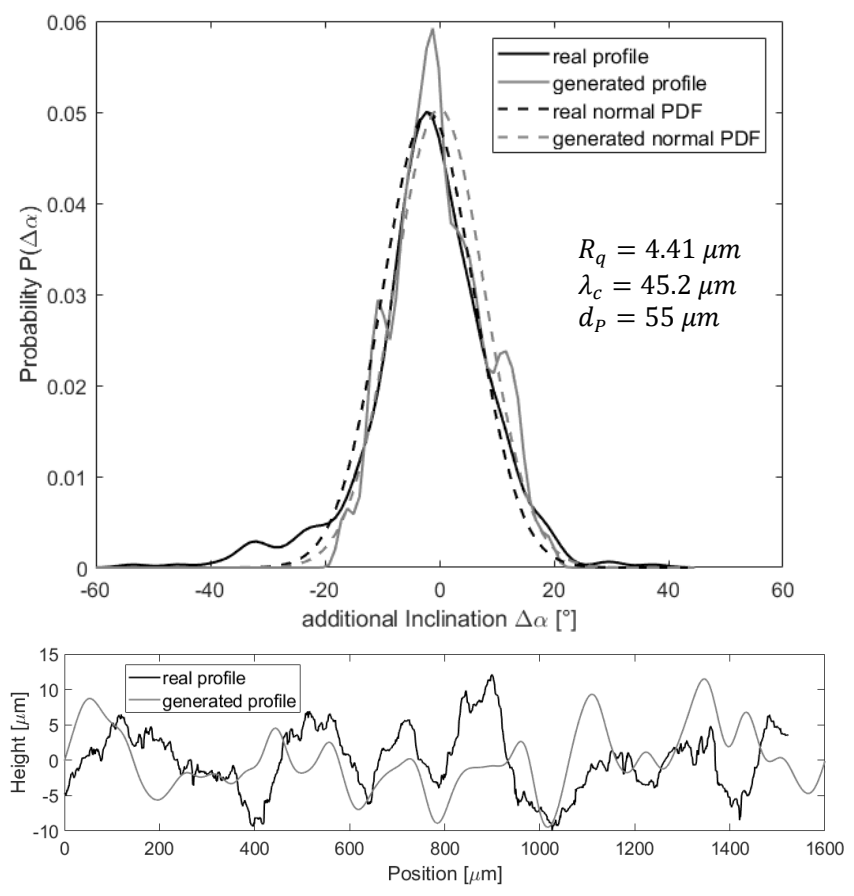

Figure 5: Roughness estimate of deteriorated compressor blade 


\section{MODELLING THE LOCAL IMPACT ANGLE}

After discussing a particle impacting normal to the surface, the variation of the global angle $\alpha_{1}$ and its effect on the inclination angle $\Delta \alpha$ is addressed. For small impact angles $\alpha_{1}$ particles may not hit the lee side of roughness structures if the absolute value of the negative slope angle becomes larger than the impact angle. This leads to a higher probability to hit the luff side of a rough structure and as a result to a shift of the probability distribution of the additional inclination angle $\Delta \alpha$ towards positive values. To evaluate this phenomena (Sommerfeld and Huber, 1999) proposed a simulation methodology (baseline) which is subject to the following three routines:

1. An additional inclination $\Delta \alpha$ is sampled from $P(\sigma, \Delta \alpha)$ as the ideal normal distribution at $90^{\circ}$ impact angle

$$
P(\Delta \alpha ; \sigma)=\frac{1}{\sqrt{2 \pi \sigma^{2}}} \exp \left(-\frac{\Delta \alpha^{2}}{2 \sigma^{2}}\right) .
$$

2. If the absolute value of a negative inclination $\Delta \alpha$ is larger than the impact angle $\alpha_{1}$, a new value of $\Delta \alpha$ is sampled to avoid a particle crossing a physical structure before hitting the surface.

3. If the generated local impact angle $\alpha_{1}^{\prime}$ would lead to a rebound towards the wall, a new value of $\Delta \alpha$ is sampled. The last step leads to the restriction that no negative inclination $\Delta \alpha$ with absolute values larger than $\alpha_{1} / 2$ is valid.

In order to avoid this time consuming iteration loop in numerical simulations (Sommerfeld and Huber, 1999) proposed that this so called "shadow effect" can be described by an effective probability distribution function

$$
P_{e f f}\left(\Delta \alpha ; \sigma, \alpha_{1}\right)=P(\Delta \alpha ; \sigma) \frac{\sin \left(\alpha_{1}+\Delta \alpha\right)}{\sin \left(\alpha_{1}\right)} \xi\left(\sigma, \alpha_{1}\right) .
$$

The term $\xi\left(\alpha_{1}, \sigma\right)$ achieves the normalization of the manipulated Probability Distribution Function (PDF). However the straight forward use of this effective PDF $P_{\text {eff }}\left(\alpha_{1}, \sigma, \Delta \alpha\right)$ might lead to unphysical results. As can be seen in Figure 6 two main issues occur: (1) The effective PDF defines a non-zero probability for local roughness angles

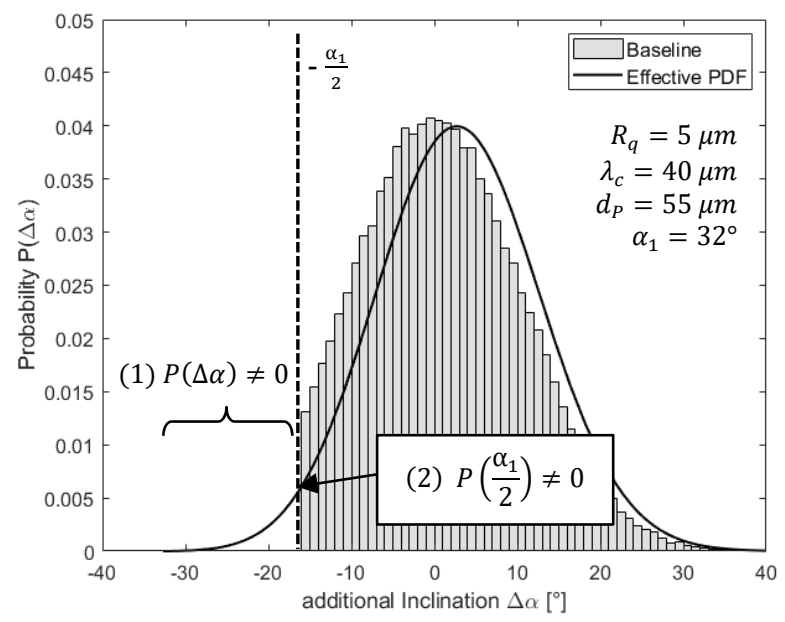

Figure 6: Approximation of the shifted distribution with the effective PDF (Eqn. (4)) smaller than the limit $\alpha_{1} / 2$. This leads to a particle rebound towards the wall. (2) Additionally the limit at $\alpha_{1} / 2$ has a nonzero probability, which represents a particle gliding on the wall after impact. This is also not possible in a physical manner, since the particle will either deposit on the wall or rebound after hitting another slope in the end (Konan et al., 2009). Based on these observations, (Konan et al., 2009) examined the possibility for a particle to make more than one rebound and the change of the rebound angle distribution because of that. As a result (Konan et al., 2009) developed a Rough Wall Multi-Collisions Model. It is based on the three routines (Sommerfeld and Huber, 1999) used to generate their validation data set (as described earlier). But instead of resampling another value of the inclination $\Delta \alpha$ in Step 2 and 3 they use an iterative stochastic driven decision routine for calculating the additional inclination angle due to multiple impacts.

In the quest to predict the shifted distribution function with higher precision than the effective PDF by (Sommerfeld and Huber, 1999) but without the effort of additional iteration routines as proposed by (Konan et al., 2009). Two modelling strategies have proven to be promising:

1. Truncated Gaussian: A modified normal Gaussian PDF defined by

$$
P_{t r}(\Delta \alpha ; \mu, \sigma, b, c)=\frac{\varphi\left(\frac{\Delta \alpha-\mu}{\sigma}\right)}{\sigma\left(\phi\left(\frac{c-\mu}{\sigma}\right)-\phi\left(\frac{b-\mu}{\sigma}\right)\right)}
$$

may be used for $b \leq \Delta \alpha$ and $P(\gamma ; \mu, \sigma, a, b)=0$ for $b>$ $\Delta \alpha$ and $c<\Delta \alpha$ (here $c \rightarrow+\infty$ ). In this context $\varphi(x)$ is the probability density function of the standard normal distribution $\left(\sigma^{2}=1\right)$

$$
\varphi(x)=\frac{1}{\sqrt{2 \pi}} \exp \left(-\frac{1}{2} x^{2}\right)
$$

and $\phi(\boldsymbol{x})$ its cumulative distribution function

$$
\phi(x)=\frac{1}{2}\left(1+\operatorname{erf}\left(\frac{x}{\sqrt{2}}\right)\right) .
$$

2. Alternatively the Weibull Distribution defined as

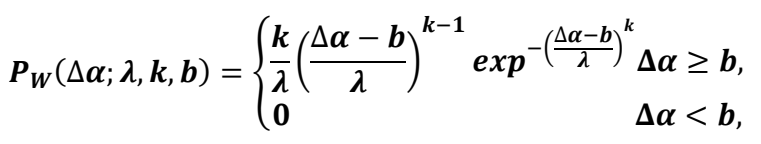

with $k\left(\alpha_{1}\right)>0$ as its shape parameter and $\lambda\left(\alpha_{1}\right)>0$ as its scale parameter might be used.

The truncated Gaussian approach can be determined by an analytical relationship from each slope angle distribution of the corresponding roughness profile. To use the Weibull distribution further pre-processing steps are necessary, because the parameters $\mathrm{k}$ and $\lambda$ depend not only on the slope angle distribution but also on the global impact angle $\alpha_{1}$. In order to minimize the amount of memory required a 


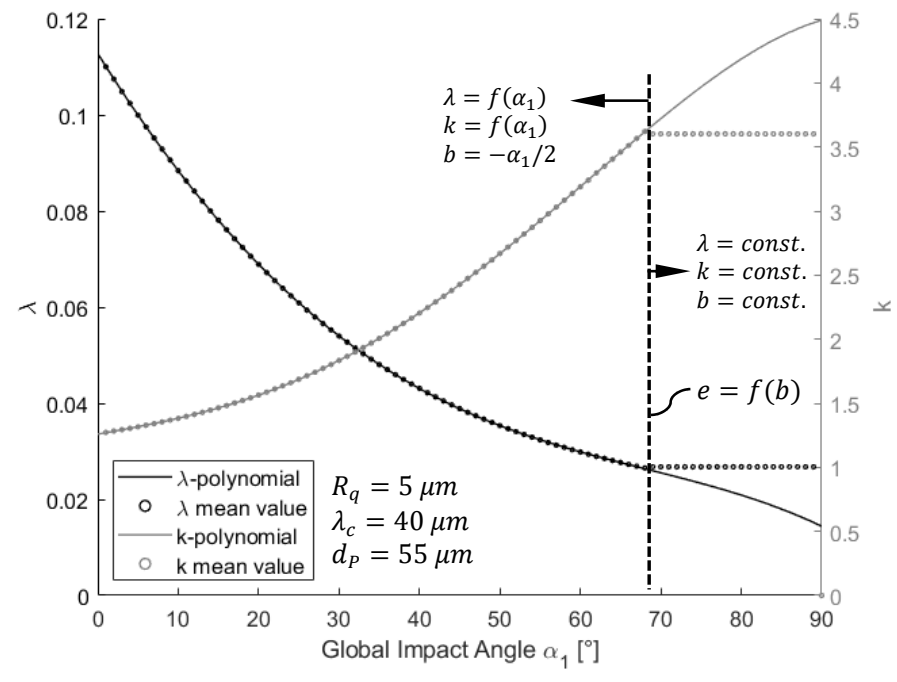

Figure 7: Polynomial approximation for $k\left(\alpha_{1}\right)$ and $\lambda\left(\alpha_{1}\right)$

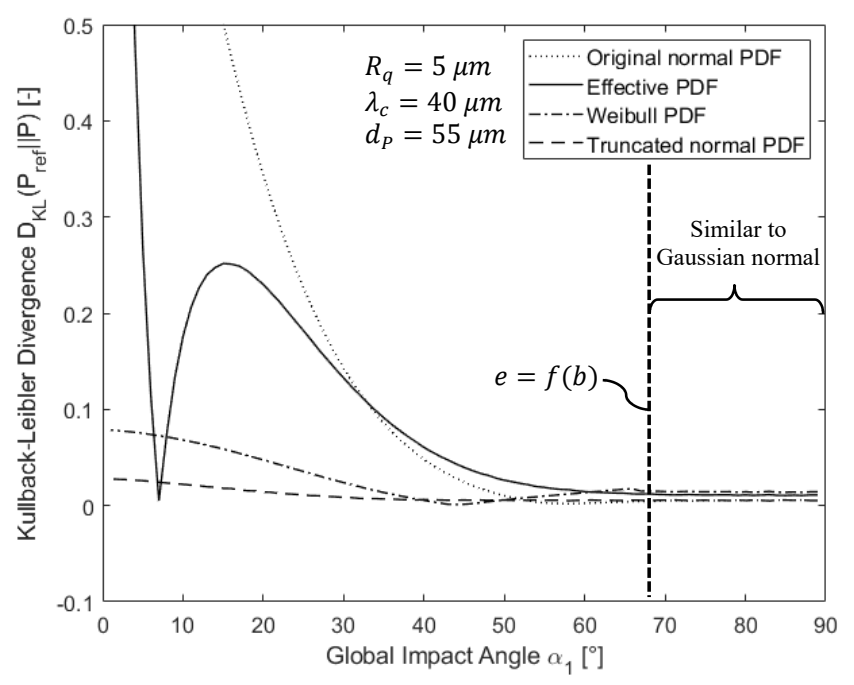

Figure 8: Kullback-Leibler Divergence $D_{K L}$ over Global Impact Angle $\alpha_{1}$ polynomial approximation of forth degree is used for $k\left(\alpha_{1}\right)$ and $\lambda\left(\alpha_{1}\right)$ instead of storing these parameters for each combination of roughness, particle size and impact angle. As can be seen in Figure 7 the polynomial can only be used up to a boundary angle e. Above e the slope angle distribution is similar to a normal Gaussian distribution. For $k=3.602$ the Weibull distribution is in good approximation equal to the normal Gaussian. Hence the parameters can be treated as constant. In order to obtain stochastic significant mean coefficient the polynomial were determined for each combination of roughness and particle diameter from a sample of 100 simulations. In each simulation the probability density functions for all global impact angles (1 to 90 in 1 degree steps) were determined using the described routine for verification purposes by (Sommerfeld and Huber, 1999). Each is based on 100.000 samples, to assure a stochastic significant sample.

In the current study, the database for the polynomial coefficients includes every combination in the course of the DoE. Constants exceeding the DoE can be determined by interpolation.

\section{CHOICE OF THE PDF}

In addition to the calculation of the coefficients the verification procedure by (Sommerfeld and Huber, 1999) enables the evaluation of the quality of the presented PDF's. The criterion to quantify the suitability is the previously described Kullback-Leibler divergence $D_{K L}$. Figure 8 shows $D_{K L}$ for the different PDF's as a function of the global impact angle $\alpha_{1}$. Examples for the compared functions at three different $\alpha_{1}$ can be found in Figure 9. Obviously the effective normal distribution is quite well suited for large impact angles $\left(D_{K L} \ll 0.1\right)$. But for small impact angles it rapidly loses quality. Here the Weibull and the truncated normal distribution are far better suited. To decide between truncated Gaussian and Weibull distribution the decision has to be made whether a slightly better global agreement with the truncated distribution for all impact angles is preferred or a physically correct mapping of the boundary areas. The truncated normal distribution has the drawback of having a non-zero probability for a sliding rebound of particles. The Weibull distribution captures this effect having a zero probability for sliding on the
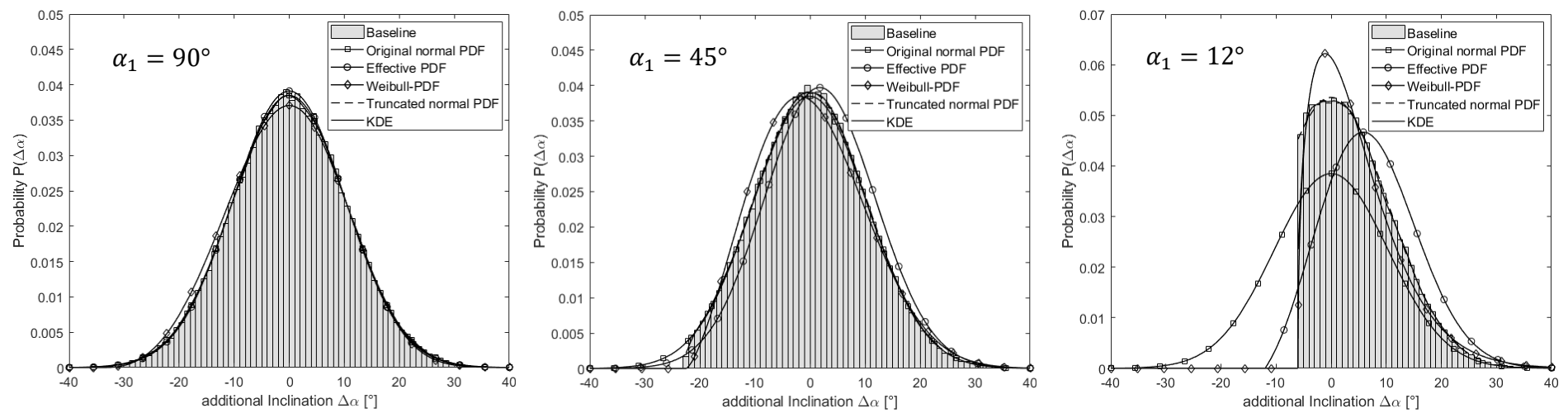

Figure 9: Additional inclination $\Delta \alpha$ for different global impact angles and $R_{q}=5 \mu m, \lambda_{c}=40 \mu m, d_{P}=55 \mu m$ 


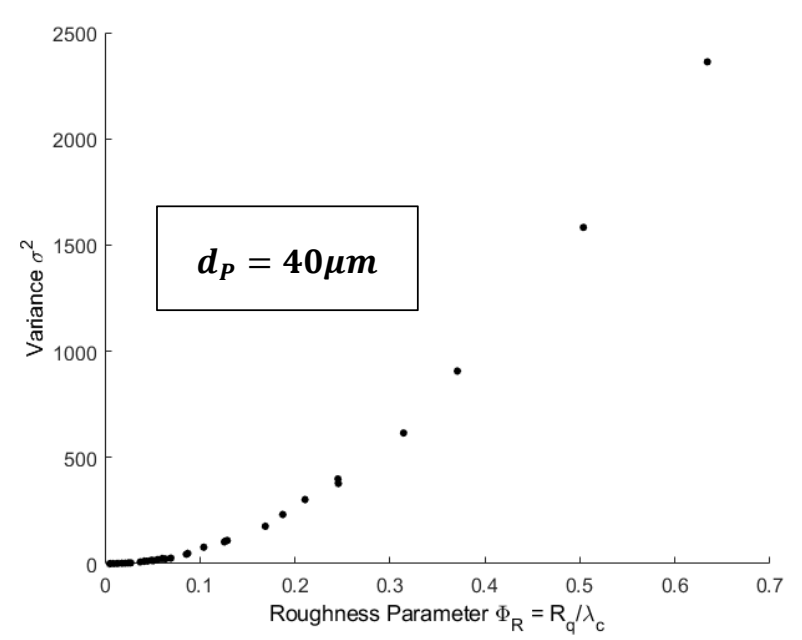

Figure 10: Variance of the additional inclination $\Delta \alpha$ over roughness parameter $\Phi_{R}$

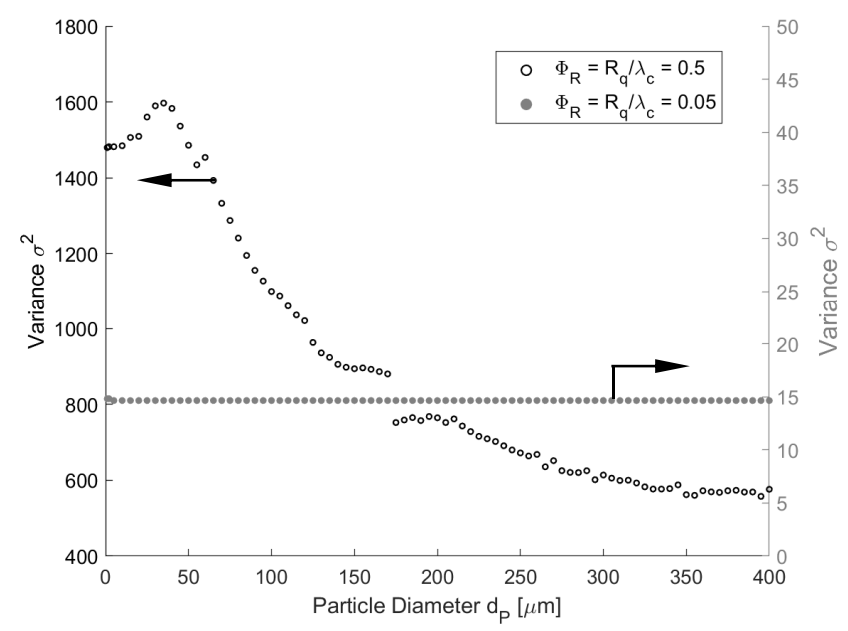

Figure 11: Variance of the additional inclination $\Delta \alpha$ over particle size $d_{p}$

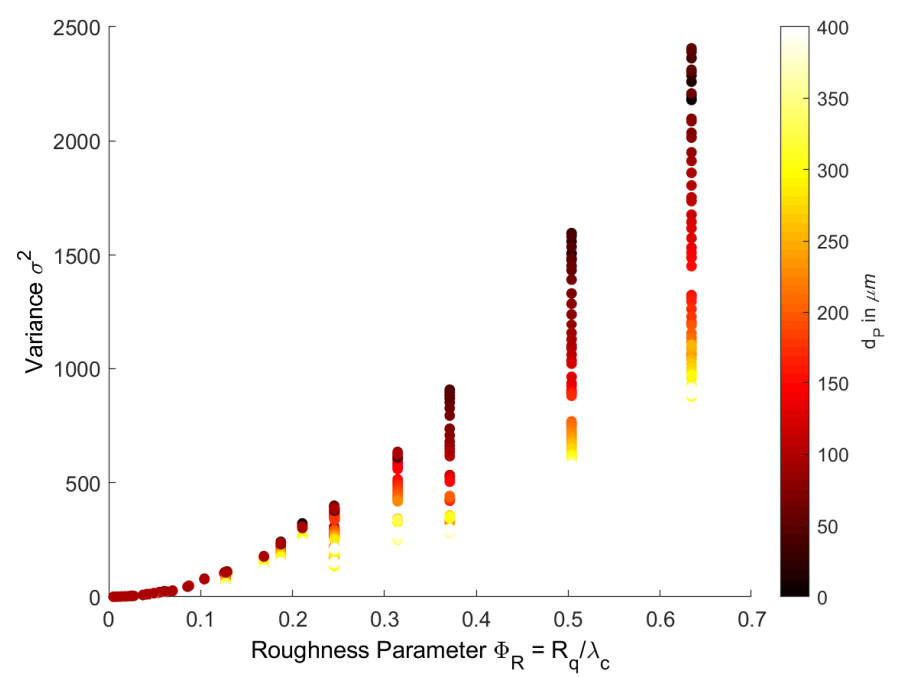

Figure 12: Variance of the additional inclination $\Delta \alpha$ over roughness parameter $\Phi_{R}$ and particle size $d_{p}$ wall. On the other hand, there is a slightly higher modelling effort for the Weibull distribution and a slightly higher $D_{K L}$. In all subsequent examples the Weibull distribution is chosen, since physically correct modelling is desired, despite the more complex calculation.

\section{RESULTS AND DISCUSSION}

Based on roughness profiles which have been found to be characteristic for performance deterioration in compressor application (Bons et al., 2001) a sensitivity study has been conducted. A dimensionless roughness parameter $\Phi_{R}$ was found that characterizes the effect of target surface roughness on statistical spread of rebound data. The roughness parameter is defined as:

$$
\Phi_{R}=R_{q} / \lambda_{c}
$$

Figure 10 shows the variance of the additional inclination $\Delta \alpha$ over the roughness parameter $\Phi_{R}$ for $d_{P}=40 \mu \mathrm{m}$. Low values of $\Phi_{R}$ correspond to small variations of the impact angles due to surface topography. Contrary higher values of $\Phi_{R}$ indicate a significant deviation between local and global impact angle. Furthermore, with increasing $\Phi_{R}$ not only the surface roughness as a single parameter but the combination of the surface topography and the particle size is significant for the variance of the inclination angle $\Delta \alpha$ (Figure 11). Hence for low values of $\Phi_{R}\left(\sim \Phi_{R}<0.1\right)$ the interaction of the particle size and $\Phi_{R}$ can be neglected during numerical simulation for simplification purposes $\left(\sigma_{\Delta \alpha}^{2}=f\left(\Phi_{R}\right)\right)$. For higher values of $\Phi_{R}$ the variance need to be evaluated with respect to the combination of particle size and roughness topography $\left(\sigma_{\Delta \alpha}^{2}=f\left(\Phi_{R}, d_{P}\right)\right)$. Figure 12 shows this dependencies for the entire parameter range of the DoE.

To evaluate the effect of the inclination angle on the statistical spread of the CoR, the developed Spread Model is connected with the Bons-Model (Bons et al., 2017) as shown schematically in Figure 2. In this process a random additional inclination angle $\Delta \alpha$ according to the probability distribution functions is generated with the principle of "inverse transform sampling" (Devroye, 1986). Let $\Delta \alpha$ be the desired parameter whose distribution can be expressed by the cumulative distribution function $\Phi_{\Delta \alpha}$. The three subsequent steps are necessary:

1. Invert the considered CDF to gain $\Phi_{\Delta \alpha}^{-1}$.

2. Generate a random number $u$ in the interval $[0,1]$ from the standard uniform distribution.

3. Compute $\Delta \alpha=\Phi_{\Delta \alpha}^{-1}(u)$.

The obtained random variable $\Delta \alpha$ fulfils the desired CDF $\Phi_{\Delta \alpha}$ and PDF. Applied to the Weibull distribution, the following dependency occurs:

$$
\Delta \alpha_{W}=\mathbf{b}+\frac{1}{\lambda}(-\ln (\boldsymbol{r n d}[0,1]))^{\frac{1}{k}}
$$

This method allows to evaluate the statistical spread in CoR data due to the surface topography. Figure 13 to Figure 16 show the synthesized CoR data for $\Phi_{R}=0.04$ and $\Phi_{R}=$ 0.13 , respectively. For this calculation particle sizes range from $1-100 \mu \mathrm{m}$, with median value of $d_{p}=10 \mu \mathrm{m}$. In addition the Bons-Model for $d_{p}=10 \mu \mathrm{m}$ is plotted as a reference. The 
theoretical variation of the surface topography leads to a significant spread in the synthesized CoR data, especially for high values of $\Phi_{R}$.

In order to analyse the observed data spread caused by surface roughness, the analytically generated data can be compared with experimentally obtained data from the literature - e.g. by Whitaker (Whitaker and Bons, 2018). The spread of synthesized data is in the same order of magnitude observed experimentally. This leads to the conclusion that the effect of surface roughness is significant for a particle's individual rebound behaviour and therefore must be taken into account in numerical simulations.

For the normal CoR the artificially generated data produce a scatter which is in good agreement with the measurement data by (Whitaker and Bons, 2018). The data spread is higher for small impact angles and decreases with rising angles. However the absolute value of the measurement data spread is under predicted for high global impact angles.

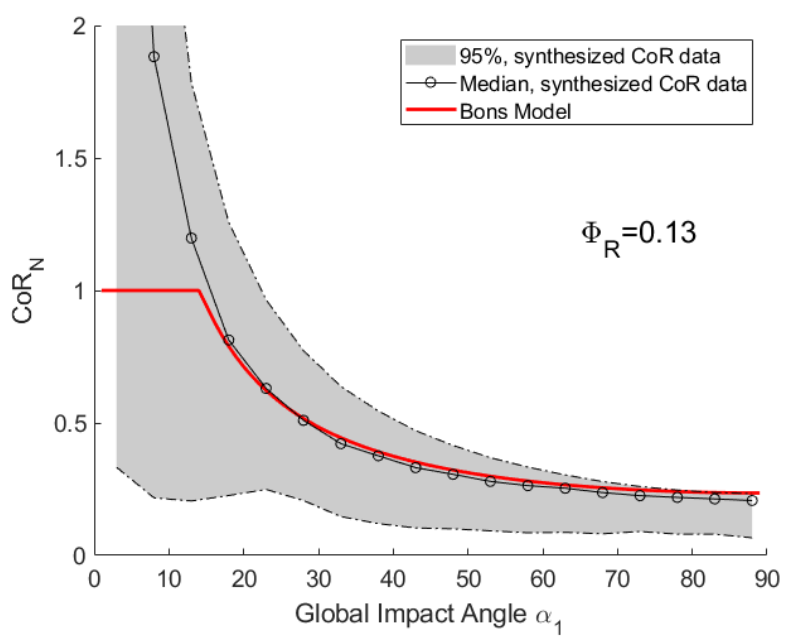

Figure 13: Synthesized $\operatorname{CoR}_{N}$ data over the impact angle in comparison to the Bons-Model

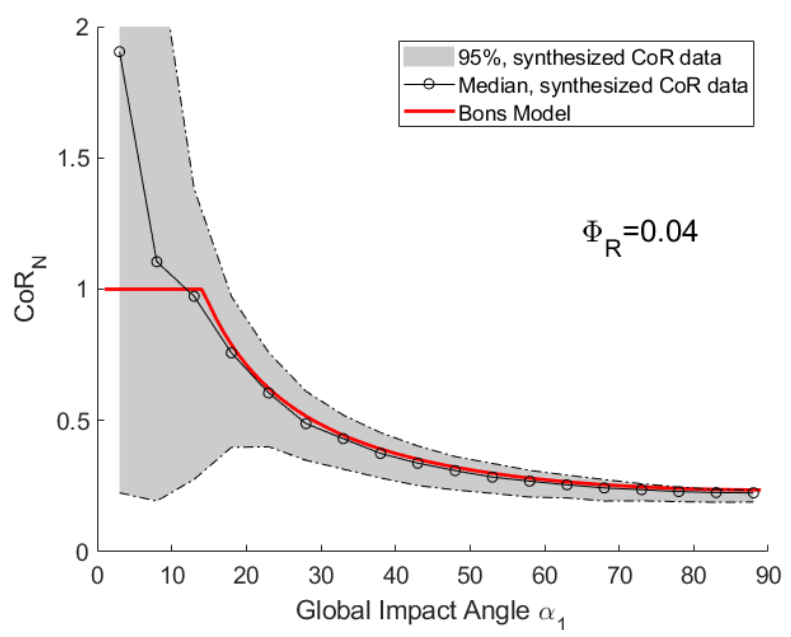

Figure 15: Synthesized $\operatorname{CoR}_{N}$ data over the impact angle in comparison to the Bons-Model
For the tangential CoR the artificially generated data produce a scatter which is in good agreement with the spread in the data by (Whitaker and Bons, 2018) up to $80^{\circ}$.

Note that the measurement data by (Whitaker and Bons, 2018) as well as the presented model predict $\operatorname{CoR}_{N}>1$ for small impact angles. At low impact angles $\alpha_{1}$, the shadow effect leads to a high probability of a significantly positive $\Delta \alpha$. The impacting particles are deflected at the rough surface and the high tangential component $v_{T 1}$ of the particle velocity is converted into a high normal fraction $v_{N 2}$ (Figure 17). Therefore $\operatorname{CoR}_{N}$ reaches values higher than unity. While the rise of normal CoR in the measured data by (Whitaker and Bons, 2018) is moderate, the shift in normal CoR values is significant higher in the prediction of the presented spread model. A similar effect can be observed for the tangential CoR for impact angles $\alpha_{1}$ close to $90^{\circ}$.

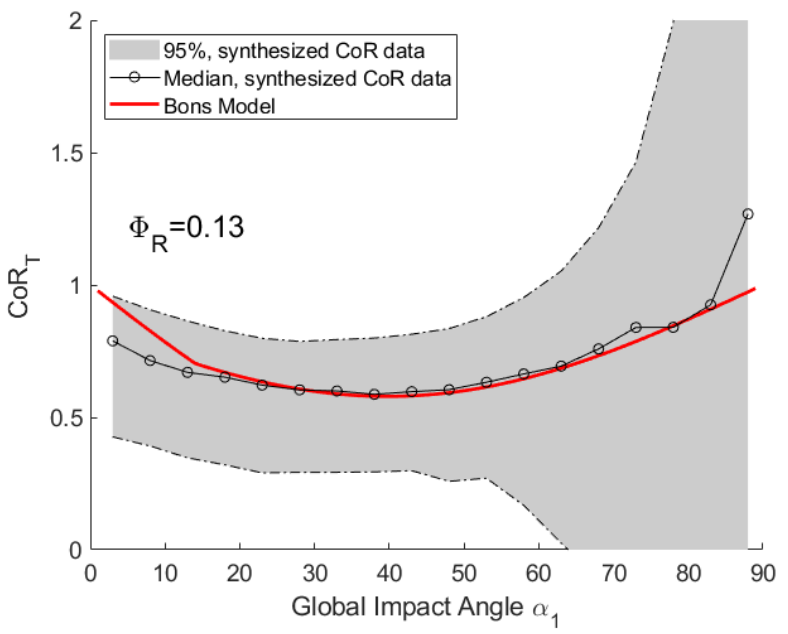

Figure 14: Synthesized $\operatorname{CoR}_{\mathrm{T}}$ data over the impact angle for in comparison to the Bons-Model

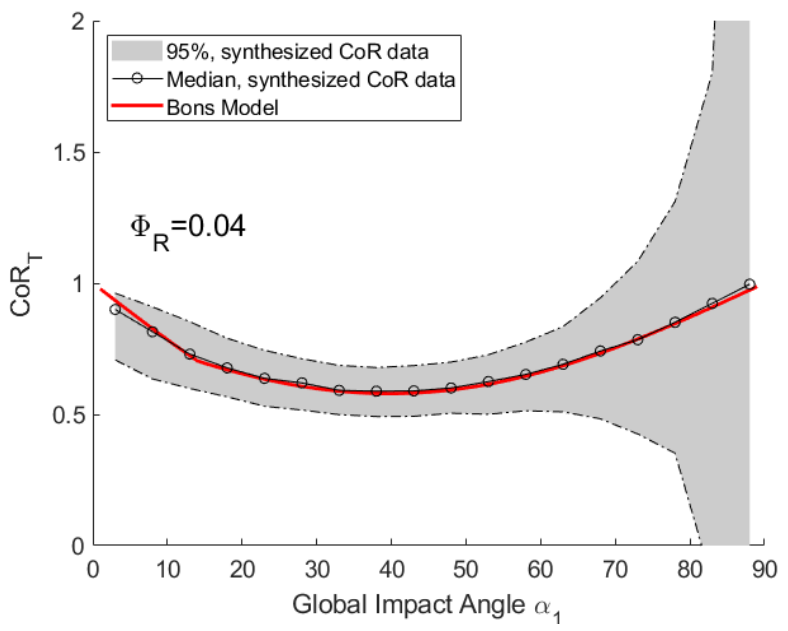

Figure 16: Synthesized $\operatorname{CoR}_{T}$ data over the impact in comparison to the Bons-Model 


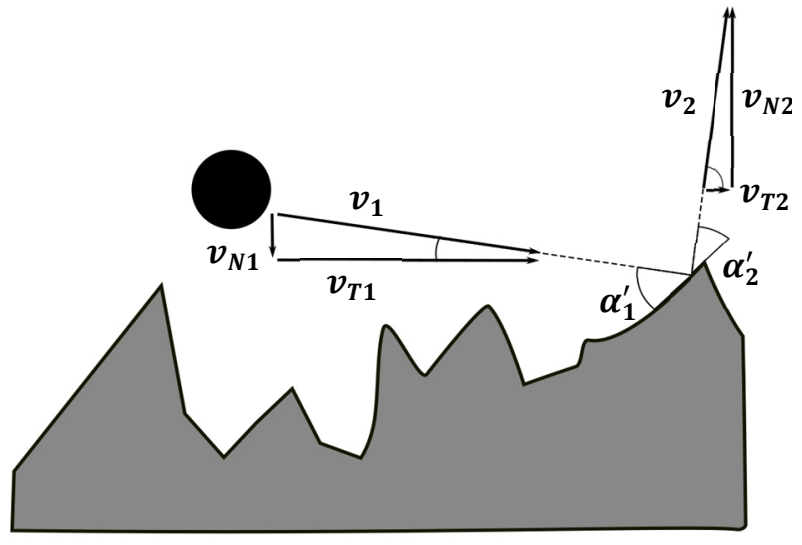

Figure 17: Particle rebound for small global impact angles $\alpha_{1}$

As can be seen in Figure 18 particles can be deflected in positive and negative direction, depending on the impact location. In addition the high normal velocity $v_{N 1}$ is converted into a high tangential fraction $v_{T 2}$. This effect was observed experimentally for positive tangential CoR. However the negative values of the tangential $\mathrm{CoR}$ are not observed in the experiment by Whitaker. This leads to the conclusion that in this region, other phenomena play a decisive role which superimpose the influence of roughness. The same applies to the discussion of the shift in normal CoR at small impact angles. Further phenomena like rolling and sliding of the particles, interaction with the surrounding fluid, or plastic deformation and erosion of the roughness peaks during contact and the associated dissipation of energy might superimpose the effect of surface roughness.

Investigation of these phenomena will be the aim of further research.

\section{CONCLUSIONS}

A spread model has been developed that estimates the statistical spread of rebound data due to target surface roughness through analytical considerations. It predicts the local impact angle of an individual particle by evaluating how deep a particle can theoretically penetrate the roughness profile with respect to its size. Based on roughness profiles which have been found to be characteristic for performance deterioration in compressor application a sensitivity study has been conducted. A dimensionless roughness parameter $\Phi_{R}$ was found that characterizes the effect of target surface roughness on rebound spread data. Low values correspond to small deviation between local and global impact angle. Contrary higher values indicate a significant deviation. Furthermore, with increasing $\Phi_{R}$ not only the surface roughness as a single parameter but the combination of the surface topography and the particle size is significant for the possible local impact angle variation that results in a spread of the coefficients of restitution. Connecting the possible

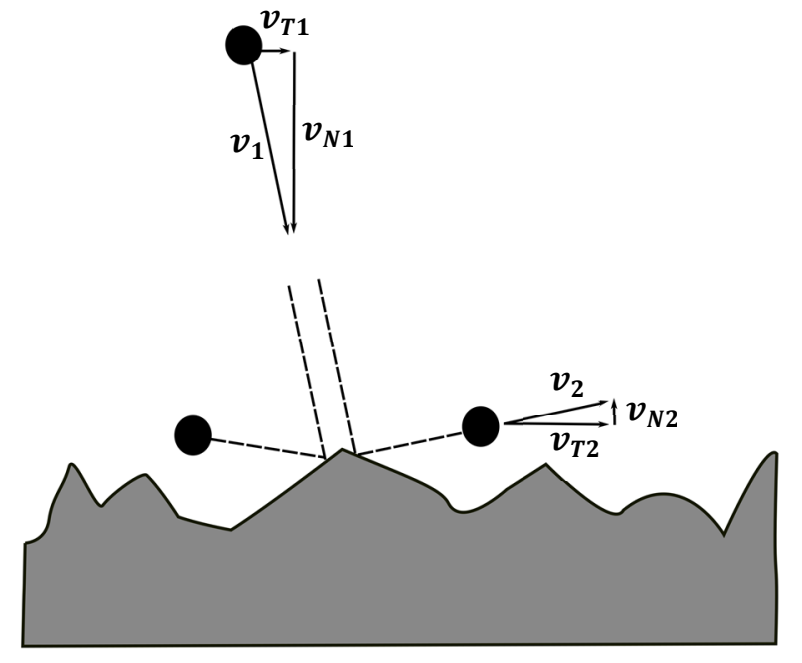
Figure 18: Particle rebound for high global impact
angles $\alpha_{1}$

variation of local impact angles with the Bons-Model induces a significant spread in the CoR-data. The spread of synthesized data is in the same order of magnitude observed by Whitaker (Whitaker and Bons, 2018) experimentally. This leads to the conclusion that the effect of surface roughness is significant for a particle's individual rebound behaviour and therefore should not be neglected in numerical simulations. This is especially relevant for increasing values of $\Phi_{R}$ and decreasing particle sizes.

\section{NOMENCLATURE}

$b \quad$ Left Boundary of Distribution

$c \quad$ Right Boundary of Distribution

$d_{P} \quad$ Particle Diameter $(\mu \mathrm{m})$

$D_{K L} \quad$ Kullback-Leibler Divergence

$e \quad$ Boundary Angle $\left({ }^{\circ}\right)$

$k \quad$ Weibull Shape Parameter

$l_{q} \quad$ Sampling length $(\mu \mathrm{m})$

$P \quad$ Probability Distribution Function

$R_{q} \quad$ Root Mean Squared of Roughness $(\mu \mathrm{m})$

$v$ Particle Velocity $(\mathrm{m} / \mathrm{s})$

$x \quad$ Random Variable

$\alpha \quad$ Global Impact Angle $\left(^{\circ}\right)$

$\alpha^{\prime} \quad$ Local Impact Angle $\left(^{\circ}\right)$

$\Delta \alpha \quad$ Additional Inclination Angle $\left({ }^{\circ}\right)$

$\varepsilon \quad$ Gaussian Random Variable

$\lambda \quad$ Weibull Scale Parameter

$\lambda_{c} \quad$ Correlation Length $(\mu \mathrm{m})$

$\mu \quad$ Expected Value

$\xi \quad$ Normalization Function

$\sigma \quad$ Standard Deviation

$\sigma^{2} \quad$ Variance

$\Phi \quad$ Cumulative Distribution Function

$\Phi_{R} \quad$ Dimensionless Roughness Parameter

$\varphi \quad$ PDF of Standard Normal Distribution 
Subscripts

$\begin{array}{cl}1 & \text { Impact Parameter } \\ 2 & \text { Rebound Parameter } \\ N & \text { Normal Component } \\ T & \text { Tangential Component } \\ v & \text { Absolute Velocity } \\ W & \text { Weibull Distribution } \\ \text { eff } & \text { Effective Distribution } \\ \text { Gauss } & \text { Gaussian Distribution } \\ r e f & \text { Reference Distribution } \\ t r & \text { Truncated Gaussian Distribution }\end{array}$

Abbreviations

$C D F$

CoR

$D o E$

erf

$K D E$

$L E$

$M C$

$M S$

$P D F$

$P S$

rnd

SS

TE

Cumulative Distribution Function

Cofficient of Restitution

Design of Experiments

Error Function

Kernel Density Estimation

Leading Edge

Midchord

Midspan

Probability Distribution Function

Pressure Side

Random Number Generation

Suction Side

Trailing Edge

\section{ACKNOWLEDGMENTS}

The project on which this report is based was sponsored by the German Federal Ministry of Education and Research (BMBF) under the funding number 01IS17051. The author is responsible for the content of this publication.

For the support in carrying out the presented studies many thanks to the student assistants affiliated to this project, especially B.Sc. J. Wallisch.

\section{REFERENCES}

Barker, B., Hsu, K., Varney, B., Boulanger, A., Hutchinson, J. and Ng, W.F. (2017), "An Experiment-Based Sticking Model for Heated Sand", in Proceedings of the ASME Turbo Exp: Turbomachinery Technical Conference and Exposition--2017, June 26-30, 2017, Charlotte, North Carolina, USA, Charlotte, North Carolina, USA, Monday 26 June 2017, ASME, New York, N.Y., V02DT48A014.

Bons, J.P., Blunt, R. and Whitaker, S. (2015), “A Comparison of Techniques for Particle Rebound Measurement in Gas Turbine Applications", in Volume 1: Aircraft Engine; Fans and Blowers; Marine, Montreal, Quebec, Canada, Monday 15 June 2015, ASME, V001T01A029.

Bons, J.P., Prenter, R. and Whitaker, S. (2017), “A Simple Physics-Based Model for Particle Rebound and Deposition in Turbomachinery", Journal of Turbomachinery, Vol. 139 No. 8, p. 81009.

Bons, J.P., Taylor, R.P., McClain, S.T. and Rivir, R.B. (2001), "The Many Faces of Turbine Surface
Roughness", Journal of Engineering for Gas Turbines and Power, Vol. 123 No. 4, p. 739.

Brach, R.M. and Dunn, P.F. (1992), “A Mathematical Model of the Impact and Adhesion of Microsphers", Aerosol Science and Technology, Vol. 16 No. 1, pp. 51-64.

Casari, N., Pinelli, M. and Suman, A. (2018), “An Innovative Approach Towards Fouling Modeling: Microscale Deposition Pattern and its Effect on the Flow Field", in Proceedings of the ASME Turbo Expo: Turbomachinery Technical Conference and Exposition--2018, Oslo, Norway, Monday 11 June 2018, ASME, New York, N.Y., V02DT47A013.

Devroye, L. (1986), Non-uniform random variate generation, Springer, New York.

Garcia, N. and Stoll, E. (1984), "Monte Carlo Calculation for Electromagnetic-Wave Scattering from Random Rough Surfaces", Physical Review Letters, Vol. 52 No. 20, pp. 1798-1801.

Kellersmann, A., Reitz, G. and Friedrichs, J. (2018), "Deterioration effects of coupled blisk blades", Journal of the Global Power and Propulsion Society, Vol. 2 No. 3, CKB8N6.

Kim, O.V. and Dunn, P.F. (2007), “A microsphere-surface impact model for implementation in computational fluid dynamics", Journal of Aerosol Science, Vol. 38 No. 5, pp. 532-549.

Konan, N.A., Kannengieser, O. and Simonin, O. (2009), "Stochastic modeling of the multiple rebound effects for particle-rough wall collisions", International journal of multiphase flow, Vol. 35 No. 10, pp. 933-945.

Kullback, S. (1978), Information theory and statistics, Reprint, Smith, Gloucester, Mass.

Richardson, J.H., Sallee, G.P. and Smakula, F.K. (1979), "Causes of high pressure compressor deterioration in service".

Serra, J. and Vincent, L. (1992), “An overview of morphological filtering”, Circuits, Systems and Signal Processing, Vol. 11 No. 1, pp. 47-108.

Singh, S. and Tafti, D. (2015), "Particle deposition model for particulate flows at high temperatures in gas turbine components", International Journal of Heat and Fluid Flow, Vol. 52, pp. 72-83.

Sommerfeld, M. and Huber, N. (1999), "Experimental analysis and modelling of particle-wall collisions", International journal of multiphase flow, Vol. 25 No. 67, pp. 1457-1489.

Sreedharan, S.S. and Tafti, D.K. (2010), “Composition Dependent Model for the Prediction of Syngas Ash Deposition With Application to a Leading Edge Turbine Vane", in ASME Turbo Expo 2010: Power for Land, Sea, and Air Volume 2: Combustion, Fuels and Emissions, Parts A and B, Glasgow, UK, June 14-18, 2010, ASME, [Place of publication not identified], pp. 615-626.

Whitaker, S.M. and Bons, J.P. (2018), “An Improved Particle Impact Model by Accounting for Rate of Strain and Stochastic Rebound", in Proceedings of the ASME Turbo Expo: Turbomachinery Technical Conference and Exposition--2018, Oslo, Norway, Monday 11 June 2018, ASME, New York, N.Y., V02DT47A016. 